\title{
Human subjects protection in the African context
}

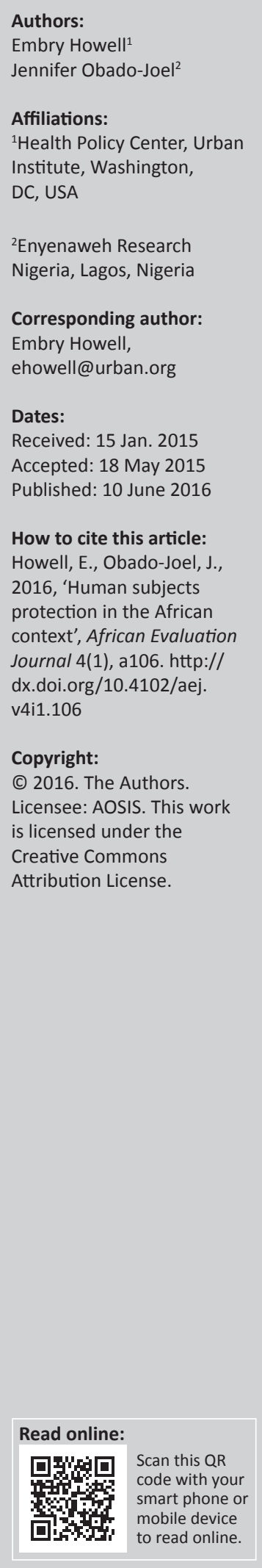

Research and evaluation are growing in Africa. All evaluators have an ethical responsibility to protect their research subjects from harm that could occur if sensitive data are revealed. In this article, we use a literature and document review to provide an overview of the protection of human subjects internationally and in Africa; we then use interviews with evaluators working in Africa to place human subjects protection principles and practice in an African context. We conclude that human subjects protection must be supported by improved guidelines tailored to the African context and local conditions; improved infrastructure for implementing and enforcing the guidelines; and increased training in awareness of human subjects principles and approaches. These efforts could stimulate increased research and evaluation and more confidence in results in the communities where research is conducted.

\section{Introduction}

One of the most critical factors in increasing accountability and knowledge growth in Africa is the need for credible statistics, research and evaluation (Data for African Development Working Group 2014; Regional Reference Strategic Framework 2006). Although the evaluation field is growing - witnessed by a growing number of papers from conferences and journals, including those presented at the African Evaluation Association meeting in Yaoundé in 2014 - continued limitations to infrastructure and human resources constrain the field (Adam et al. 2011). One frequently overlooked concern with this development is that appropriate human subjects protection systems have not kept pace with the increase in the number of studies underway.

Protection of human subjects requires that no sensitive information be revealed outside the confines of a research or evaluation study, using the 'need-to-know' principle. The need-to-know principle states that each member of the evaluation team should only know the identity of individuals when absolutely necessary for their role in the conduct of the study. Such protection is most important when access to information by unauthorised persons could do harm, for example when there is potentially stigmatising information (e.g. orphanhood, HIV status) that could lead to exclusion from a community, school, benefits or jobs.

Recognising that there is a growing need for human subjects protection in African evaluation research, this article reviews the principles of human subjects protection and the status of their implementation in Africa. Drawing on literature, documents and the opinions of 12 evaluators working in Africa (eight from the United States and four who are African), we seek to uncover continued gaps and barriers in order to foster further improvement in human subjects protection in African evaluations. The convenience sample of evaluators is not a statistically representative sample, but has rather been chosen to convey additional qualitative insights to provide further understanding of the African context.

\section{Background}

A human subject - a term used frequently in this paper - is defined as 'a person from whom or about whom you collect information during your assessment or evaluation' (Presidential Commission for the Study of Bioethical Issues 2011). A person becomes a subject of a study in a variety of ways, for example by being interviewed or observed. Two closely related concepts are privacy and confidentiality. Privacy is being free from being observed or disturbed by other people without consent, while confidentiality is guarding information related to an individual unless the individual gives consent to its disclosure (Duncan, Elliot \& Juan Jose Salazar 2011). Within the context of broader human rights ethics, the protection of privacy and confidentiality of personal information is a universal human right. However, the protection inevitably must be contextspecific in terms of how procedures are implemented (Jeko, Mangwaya \& Blignaut 2012). 
Previous studies have pointed to the need for improved human subjects protection in Africa. For example, a 2000 survey of 203 health researchers in developing countries ( 59 from Africa) found that about a quarter of the studies by these researchers had not received a review of human subjects protection (Hyder et al. 2004). In 2003, the WHO African Regional Office found that $36 \%$ of member countries had no formal human subjects review process and that limited resources affected the quality of review in many other countries (Kirigia, Wambebe \& Baba 2005). However, two other studies covering the period 2000-2012 found that an increased focus on research ethics and training had improved health research ethics capacity in many places in Africa over the past decade (Ali, Hyder \& Kass 2012; Ndebele et al. 2014).

Still, there is much room for sustained focus on this issue. For example, the following types of barriers to adequate human subjects protection were enumerated at a training conference on research ethics in Swaziland in 2013 (Council on Health Research for Development 2013):

- Lack of a legal framework and guidelines for human subjects review.

- Lack of research ethics review capacity, particularly for the non-scientific research and evaluation community.

- Lack of resources, both human resources and infrastructure (e.g. office space and computers).

- Need for basic and continuous training in research ethics.

Three important historical events led to the current ethical framework for human subjects protection. The Nuremburg Code, resulting from Nazi war crime trials, established the principal of informed consent, whereby research subjects should be informed of the purpose of the study, their right to decline participation and any risks of harm to them (Weindling 2001). Another landmark case on the protection of human subjects was the Tuskegee study of black American men between 1932 and 1972 in the American South (Centers for Disease Control and Prevention 2014). Subjects of the study who had syphilis were placed in a treatment or a control group without any knowledge of the study. The control group was not given the treatment, even though it was known to be effective.

The Belmont Report (National Commission for the Protection of Human Subjects of Biomedical and Behavioral Research 1979) laid the foundation for basic ethics in research studies involving human subjects. The Belmont Report outlined three main principles for ethical research. These include the following:

Respect for persons: Special protection should be guaranteed for individuals with diminished autonomy.

Beneficence: Persons should be treated with consideration and respect by acknowledging their rights and voice during the course of the study. Individuals participating in studies are to be protected from harm, with guaranteed protection of their well-being, maximizing possible benefits and minimizing potential harm to them.
Justice: Persons participating in studies should not be targeted based on any form of economic, racial or social disadvantage. Equity must be maintained in selection of research subjects by ensuring participants are selected primarily based on their relationship to the subject matter.

The ethical principles outlined in the Belmont Report are generally accepted worldwide and are consistent with the United Nations Universal Declaration of Human Rights (United Nations 1948), which was signed by 48 nations, including three in Africa. (Most were still under colonial rule at the time.) Article 1 of the Declaration states that all human beings are born free and equal in dignity and rights; no one shall be subjected to arbitrary interference with his or her privacy. Article 12 further states that everyone has a right to protection of the law against such interference. The Helsinki Principles for research using human subjects in medical studies (World Medical Association 2008) further the same ethical principles and have been widely disseminated by the World Medical Association. ${ }^{1}$

One core principle of human subjects protection is informed consent. The serious ethical breaches described in the examples above from Nuremburg and Tuskegee led to the generally accepted principle of informed consent, whereby individuals participating in research and evaluation studies should be informed of any risks of harm as well as the benefits of the study, with the ability to decline participation. For consent to be 'informed', the individual must be of the age of accountability and with full mental capacity to make judgments. Informed consent by children and individuals with mental health issues should be given by proxy. Specifically, the type of information that should be disclosed to a study participant is shown in Box 1.

Yet, in spite of this generally accepted ethical framework, the implementation of these ethical principles is certainly not uniform around the globe.

One way to achieve more uniformity in human subject protection is to establish a legal and regulatory framework within each country. This framework may be established either through national legislation or through regulation pertaining to a broader law.

\section{US-funded research ethics guidelines}

US-funded research and evaluation conducted either in the United States or abroad must conform to both US and host country regulations. Examples of such studies include research and evaluation studies funded by the US National Institutes of Health or the Centers for Disease Control and Prevention.

In the United States (and across the world), regulations on human subjects protection have focused foremost on health

1.African members of the World Medical Association are Angola, Cameroon, Cape Verde, Cote D'Ivoire, Democratic Republic of Congo, Egypt, Ghana, Somalia, South Africa, Sudan, Tanzania, Tunisia and Uganda. 
BOX 1: Ethical guidelines for disclosure of study information for informed consent.

1. Purpose of the study

2. Risks to the individual

3. Potential benefits to the individual or others

4. Alternatives to the research protocol

5. Confidentiality protections

6. Compensation in case of injury

7. Contact information for questions

8. Conditions of participation, including right to refuse or withdraw

Source: Office for Human Research Protections, U.S. Department of Health and Human Services, 2014, International compilation of human research standards, viewed 6 January 2015, from http://www.hhs.gov/ohrp/international/intlcompilation/2014intlcomp.pdf

research and evaluation, which is where the most egregious ethical breaches have occurred. For example, global protocols for protection of human subjects in clinical trials have been issued by the Council for International Organizations of Medical Sciences (2002). However, there is no comparable global regulatory framework for human subjects protection in the behavioural sciences or other disciplines. Consequently, such studies may or may not receive an ethics review.

The work of institutional review boards - generally called research ethics committees (RECs) in Africa - serve as the main enforcement mechanism for overseeing the implementation of human subjects protection in the United States. Evaluators may be required by their funder or institution to submit, or may voluntarily submit, a study protocol for review. Expedited review may waive some review requirements, but studies suitable for expedited reviews must pose minimal risks to participants. For example, reviews for studies with vulnerable populations are generally not expedited. For each study reviewed, the committee must consider the following:

- Risks to human subjects.

- Adequacy of protection against these risks.

- Potential benefits of the research to the subjects and others.

- Importance of the knowledge to be gained, which entails a consideration of the validity of the research methods.

Once established by an institution (for example a government agency such as the National Institutes of Health or a university), the committee may or may not review all research within the institution, or may concentrate on one type of study, most often health-related studies. A study with full or partial US government funding usually requires review by a committee registered with the US Department of Health and Human Services. However, a concern for human subjects protection in the United States, and more generally in all countries, is that this regulatory framework is not as pervasive for research and evaluation studies in non-university settings or for non-health-related research and evaluation. In addition, the work of government statistical agencies or ongoing 'monitoring and evaluation' data collection is often exempt from review. These variations lead to variations in how human subjects protection is implemented. Protecting human subjects in non-reviewed studies is more challenging, because it relies primarily on the intuition and ethical sensitivity of the evaluator. For example, the protection should be just as strong for evaluation of a programme to improve the education of street children as for a clinical trial of a drug.
However, the legal and regulatory framework for human subjects protection in the first study might not exist in the United States, as well as abroad.

\section{The African context}

Human subjects protection is still developing in many African countries. This is due to a lack of legal and regulatory infrastructure, a lack of enforcement mechanisms for such guidelines and other practical considerations. In particular, protection may be compromised by poor infrastructure (for example, a lack of locked storage or secure computers) or other factors, such as language or cultural barriers. This becomes a particular concern with the growing body of evaluation studies on sensitive topics such as HIV / AIDS and mental health.

\section{Regulatory frameworks}

Research ethics regulations exist to ensure that human subjects protection is not left solely to the personal morality of individual researchers. A legal framework for human subjects protection in many African countries emerged from post-colonial legislation protecting human rights. Laws and regulations vary substantially across the continent, both in content and the agencies responsible for overseeing their implementation. Table 1 shows a list of 15 selected African countries, the effective date of their ethics law or regulation and the implementing agency. Countries with the earliest legislation for protecting human subjects include Botswana (1967), Malawi (1974) and Kenya (1979). As shown, sometimes there are multiple agencies involved in overseeing human subjects protection (e.g. Cameroon, Ethiopia and South Africa) and sometimes only a single health agency is involved (e.g. Nigeria).

According to the evaluators with whom we spoke, from their personal experience the current regulatory framework is uneven across Africa, both in the availability and content of guidelines and the degree of enforcement of guidelines. They note that Kenya and South Africa have the strictest and most uniformly enforced guidelines, but this is generally within the confines of health-related academic studies. For example, much of the data collected for monitoring and evaluation studies - such as the individual-level data required for the monitoring of the President's Emergency Plan for AIDS Relief $(\text { PEPFAR })^{2}$ programme - falls outside the human subject protection guidelines. Consequently, there is no regulatory framework that requires informed consent or protection of privacy and confidentiality in such evaluation studies.

Another limitation to human subject protection in Africa is the lack of capacity to enforce regulations through a network of RECs. Researchers note that the advantage of a REC review is that it helps a researcher focus more carefully on human subject protection guidelines as they undertake research and evaluation studies.

2.President's Emergency Plan for AIDS Relief, a US government-funded programme to provide HIV prevention and treatment in selected African countries and other places around the world. 
TABLE 1: Research ethics regulations in 15 African countries.

\begin{tabular}{|c|c|c|}
\hline Country & Date of law/regulation & Implementation agency \\
\hline Botswana & 1967 & $\begin{array}{l}\text { Health Research and Development Committee and the Health Research Unit } \\
\text { of the Ministry of Health. }\end{array}$ \\
\hline Cameroon & 1987 & Ministry for Scientific Research, Ministry of Health. \\
\hline Egypt & 2003 & Central Directorate for Research and Health Development. \\
\hline Ethiopia & 1999 & Ethiopian Science and Technology Commission, Ethiopian Health Department. \\
\hline Ghana & No national regulations; use international guidelines & Ghana Health Service and Ghana Ministry of Education. \\
\hline Kenya & 1979 & $\begin{array}{l}\text { National Council for Science and Technology, Ministry of Health, Kenya } \\
\text { Medical Research Institute. }\end{array}$ \\
\hline Malawi & 1999 & $\begin{array}{l}\text { National Research Council of Malawi; sectoral ministries encouraged to } \\
\text { develop own guidelines. }\end{array}$ \\
\hline Mozambique & 1974 & Science and Technology Ethics Code applies to all sectors. \\
\hline Nigeria & 2007 & National Health Research Ethics Committee. \\
\hline Rwanda & 2009 & Ministry of Health/National Ethics Committee. \\
\hline South Africa & 2003 & $\begin{array}{l}\text { National Health Research Ethics Council, National Health Research } \\
\text { Committee. }\end{array}$ \\
\hline Sudan & 2008 & Ministry of Health. \\
\hline Uganda & 2007 & National Council for Science and Technology. \\
\hline Zimbabwe & 2011 & Medical Council of Zimbabwe. \\
\hline
\end{tabular}

Sources: Harvard Global Ethics Research Map (https://webapps.sph.harvard.edu/live/gremap/view.cfm) and the International Compilation of Human Research Standards 2014 Edition. http://www. researchethicsweb.org/hrweb/

The REC review can also serve as a means for regular monitoring of privacy and confidentiality practices, depending on the enforcement mechanisms, such as review of consent forms and requirements for regular reports to the committee.

However, many African countries have no REC within the country, and thus even when guidelines exist there is no enforcement mechanism for human subjects protection. In other countries there may be only one such committee, which may be overburdened. Researchers working in countries with no RECs or no research ethics guidelines report that they must rely on their own personal and professional ethics. This is another great source of variability in human subject protection across the continent.

Still, large numbers of RECs do exist and capacity is growing. One study found over 155 RECs operating across Africa, but with a great variability in their skills, membership and efficiency (AAREC 2011). A study on training needs by RECs in Cameroon, Mali and Tanzania found that membership of RECs in these countries ranged between 48 and 150 people (Ateudjieu et al. 2010).

Table 2 also shows the number of ethics review committees for 18 African countries. The number ranges from 10 or more RECs in Egypt, South Africa and Uganda to very few or a single committee in many countries. The number of RECs may not be reflective of the comprehensiveness of the review process. Kenya, with its multi-tiered REC process, is perhaps the most comprehensive review on the continent due to the existence of the Kenya Medical Research Institute (KEMRI) and the National Council for Science and Technology, which conducts much of the ethical review in the country and has adequate resources and experience to do so:

I have found KEMRI to be increasingly rigorous in their ethical review over the past 8 years; which is great - it means better protection for patient privacy and confidentiality and improved
TABLE 2: Number of research ethics committees in 18 African countries.

\begin{tabular}{ll}
\hline Number of RECs & Countries \\
\hline 1 & Central African Republic, Morocco and Namibia \\
$2-4$ & $\begin{array}{l}\text { Algeria, Botswana, Gabon, Kenya, Malawi, Rwanda, Tunisia, } \\
\text { Zambia and Zimbabwe }\end{array}$ \\
$\begin{array}{ll}5-9 & \text { Ethiopia, Cameroon and Democratic Republic of Congo } \\
10+ & \text { Egypt, South Africa and Uganda }\end{array}$ \\
$\begin{array}{ll}\text { Source: } \\
\text { researchethicsweb.org). RECs, research ethics committees }\end{array}$
\end{tabular}
Source: Ijsselmuiden et al. (2012), Mapping Ethics
researchethicsweb.org). RECs, research ethics committees

oversight, accountability, and awareness about ethical issues among staff working on the project. (Quote from a U.S. researcher working in Kenya)

An additional factor in many places is that even when a REC exists, it may not meet regularly or may not have adequate administrative support to function well due to a lack of resources for the committee. For example, one study of 12 RECs in Africa found that only five had any paid staff, and six had no operating budget at all (Kass et al. 2007). This under-resourcing leads to bottlenecks in the number of studies that can be reviewed, increasing the time needed to obtain ethical review and limiting the scope of research and evaluation that can be considered (Council on Health Research for Development 2008; Annual National Research Ethics Conference 2013). A contributor to evaluators choosing to avoid ethical review (particularly when it is not required) is the high charge that many committees now place for review, sometimes more than $\$ 1000$ per study. This could lead to only studies being reviewed that have sufficient funding to pay for review, certainly not a good result for widely improving human subjects protection.

Another deterrent is the time period that it may take from submitting a request for review until receiving approval. Evaluators noted that this could take up to a year or more, particularly for an overburdened committee. The study may be under a strict time requirement, so the evaluators may proceed with project tasks while waiting for approval, leading to inconsistencies in procedures: 
'There have been times when we have gone ahead and collected the data without first having the research reviewed and approved. If the data captured is not already in our RECapproved protocol then the methods may have not been in full compliance with the protocol.' (Motswana researcher working in Botswana.)

There are also intergovernmental coordination challenges in human subject protection. This is quite evident in de-concentrated governance systems in which the responsibility for ethics monitoring differs at the national and subnational levels. For example, in Nigeria, ethics review guidelines and infrastructure exists at the national level and sometimes at the state level. However, RECs are almost absent at the local government level, where many studies are conducted. On the other hand, unless there is reciprocity for accepting the review of a local REC, the national government might require an additional review. Such a study might be faced with the need to do multiple reviews - for example by the funder, the national government and the local government - further increasing the time and cost of a study.

\section{Informed consent}

A hallmark of human subject protection is the need for informed consent, whereby individuals are informed of the risks and benefits of a study and allowed to agree or not agree to participate. Obtaining adequate informed consent is very challenging in an African context.

Culture and its influences permeate all forms of social interaction in African societies. Although legal regulations are of value in enforcing ethical practices, researchers and evaluators must adapt those practices to the local cultural context where the research is conducted. This is especially true in obtaining informed consent. Even though human subject guidelines often are adapted from international guidelines, there is a question about whether informed consent guidelines from Western contexts are appropriate for many African settings. Hugman, Pittaway and Bartolomei (2011) argue that violating cultural norms by agreeing to participate in an evaluation study could lead to the unintended consequence of social exclusion or worse.

Further, given how difficult it is to conduct evaluations, these kinds of restrictions, without appropriate adaptations, may severely restrict who voluntarily participates in a research study, impeding the conduct of the study. Even though women are of legal age to give consent according to international guidelines, they may not be allowed to do so individually in some contexts. In certain cultures, women are not permitted to speak to strangers without the presence of another family member, and male researchers may be prohibited from communicating with the women in a household. Although cognitive ability is generally the guideline for informed consent, in this circumstance cognitive ability would not be a sufficient argument for requesting consent. This presents a severe challenge if the topic is a sensitive one such as reproductive health or family violence.
Another example pertains to obtaining consent from adolescents. African adolescents may function as adults, either as young brides living independently in urban areas or as culturally recognized heads of family. Yet human subject guidelines often require that parents or guardians give consent for them. In many African societies, discussion of sexuality between youth and adults is not allowed. Consequently, involving parents or guardians in the consent process may exclude at-risk young people from reproductive and sexual health studies, even though they would consent to the research as individuals (Zuch et al. 2012).

Low literacy is also a major challenge for informed consent. An illiterate person cannot personally read materials on the study and sign a consent form. One evaluator explained that, in a study in Tanzania, he had to depend on verbal consent from the respondents (personal communication with a US researcher working in Tanzania). The verbal assent to the study was then entered into a form and was witnessed. Alternatively, in other evaluation studies a signature may be in the form of a thumbprint. Because witnesses may be community members and the research subject may be from a vulnerable group (e.g. HIV-positive individuals), it is critical that the witnesses be selected with care to assure confidentiality.

\section{Privacy and confidentiality}

A common challenge reported by all our interview respondents was protection of privacy and the confidentiality of research participants' information in low resource settings. This concerns all levels of data collection and storage.

Firstly, there may be very limited privacy when conducting interviews. For example, interviews may occur in a house, village, public clinic or other public space, where there is no private space for the interview. In such a setting, the interview may be overheard by an interested family member, neighbour or colleague. One evaluator reported finding out about the HIV status of someone from the salesperson at a neighbourhood store; apparently the storekeeper overheard a conversation at the local clinic due to a lack of privacy at the facility (personal communication with a Nigerian researcher working in Nigeria).

In addition, often the data are collected in paper records and there may be no locked file storage for files, especially in rural or other under-resourced settings. Interviewers may carry the files around the community or to their home, further risking exposure of the data. Once the study is over, data may be held for a long time in such insecure settings, further exposing research subjects to harm.

Once the data are automated, there are additional challenges, particularly with sharing data across members of the research team or from organization to organization. For example, one evaluator highlighted the challenges in transmitting data securely while working on research projects in rural communities with scant access to electricity, telephone lines 
or the Internet (personal communication with a US researcher working in South Africa). There may be no secure means of transmission at all. Increasingly in such situations, data may be collected using small digital devices and stored on memory sticks and other electronic media. Such devices are in high demand and are frequently stolen. Once lost or stolen, the data may be accessible to those outside the research team if not carefully password-protected. Memory sticks may be subject to viruses that destroy or corrupt the data, and there may be no facility for backing up data. It is understandable why evaluators may retain multiple copies of data in these circumstances, but this creates the risk of further improper harmful disclosure. Password protection is critical for data protection when data are transmitted, but it is not universally used.

\section{Awareness of the need for human subjects protection}

Because of a lack of sufficient regulatory frameworks and enforcement of guidelines, African researchers and evaluators often must rely on their own ethical framework and experience in similar circumstances. Evaluators expressed that, although there is a substantial amount of training available to African researchers and evaluators, there is still a need for more awareness of human subjects protection, particularly at the practical, local level.

When asked about awareness in the field, two researchers gave slightly different opinions:

'In general, there is not a good level of awareness of data security and confidentiality.' (Evaluator from Botswana working in Botswana)

'Researchers are aware of these things, but the practical application of these guidelines is not necessarily understood ... The challenges are in providing practical application of the information during training. Ideally, they would have the chance to write a consent form, apply it while collecting data outside the classroom, return to store and enter the data, and then process de-identified data.' (US evaluator working in Tanzania)

This suggests the need for more training to reinforce both awareness as well as the practical day-to-day processes that will be needed to protect human subjects in Africa. Unfortunately, while there are many online courses, these usually are not designed to provide guidance that is specialized to the African context. In addition, taking such courses online may require Internet connections with a speed that is not available many places in Africa, which calls for more in-person training.

\section{Conclusions and recommendations}

Across Africa, evaluators face many challenges in protecting those who participate in their studies. Firstly, when regulatory frameworks and guidelines exist (which is more often for health research), the guidelines often have not been targeted to the special circumstances in many local communities. In addition, in many countries, REC review (used to enforce guidelines) is not widely available and is uneven in quality.
The Kenyan multi-tier REC process is a good model that could be replicated by other African countries as a starting point in improving human subject protection. An additional benefit of such a regularized process is that countries such as Kenya with a robust REC framework have developed a more vibrant research environment that fosters evaluation.

Evaluators working in countries without such guidelines or on studies without REC review must use their own ethical principles and judgment, such as using kindness, diplomacy and respect for all their research subjects. Given variations in these qualities across evaluators, this undoubtedly has led to an uneven application of the principles of human subject protection (a situation that is certainly not unique to Africa). Thus, because protecting human subjects relies on the awareness of evaluators, it is important to include training and retraining of researchers as a key component of any evaluation, regardless of the regulatory framework or REC requirements. This could be built into any evaluation project, as a part of overall training on research processes.

There are also many practical challenges to enforcing human subject protection, especially in low resource settings. Obtaining informed consent, a critical step in protecting human subjects, can be difficult. For example, protecting privacy and confidentiality was reported as a huge concern for African evaluators. Factors include the lack of secure computer systems and other data storage facilities and a lack of a secure means for transmitting data from the field. Solutions to this challenge require additional resources devoted to data security, such as budgeting for locked storage and secure computer systems. Confidentiality is also jeopardized when data are collected in rural or crowded urban settings, because of the absence of a private space for interviews. Evaluators must continually monitor their research environment closely, for example to ensure there is adequate space between the survey respondents and other people.

Challenges with informed consent include a low level of literacy, as well as cultural or religious norms that determine who can give consent. Thus, consent forms and processes must be culturally sensitive and be written and phrased in languages understood by participants. Such an approach will have the dual effect of building confidence in research and therefore increasing the number of willing research subjects. In turn, increased confidence from study participants will help researchers collect more accurate and in-depth information.

In summary, human subjects protection must be supported by improved guidelines tailored to the African context and local conditions; improved infrastructure for implementing and enforcing the guidelines; and increased training in awareness of human subject principles and approaches. These efforts could stimulate increased research and evaluation and more confidence of results in the communities where research is conducted. 


\section{Acknowledgements Competing interests}

The authors declare that they have no financial or personal relationships that may have inappropriately influenced them in writing this article.

\section{Authors' contributions}

E.H. was the project leader and conceived the research. She drafted portions of the original manuscript and edited the final manuscript. J.O-J. conducted interviews, drafted portions of the manuscript and prepared the tables.

\section{References}

AAREC, 2011, 'Report on First African Conference for Administrators of Research Ethics Committees', viewed 3 March 2016, from https://www. healthresearchweb. org/files/AARECFinalReport.pdf

Adam, T., Ahmad, S., Bigdeli, M., Ghaffar, A. \& Røttingen, J.A., 2011, 'Trends in health policy and systems research over the past decade: Still too little capacity in low-income countries', PLOS One 6(11), E27263. http://dx.doi.o/rg10.1371/journal.pone.0027263

Ali, J., Hyder, A. \& Kass, N., 2012, 'Research ethics capacity development in Africa: Exploring a model for individual success', Developing World Bioethics 12(2), 55-62.

Annual National Research Ethics Conference, 2013, 'Proceedings of the 5th Annual National Research Ethics Conference', viewed 12 October 2014, from http://www. uncst.go.ug/dmdocuments/ANREC 5 proceedings booklet 2014 with cover.pdf

Ateudjieu, J., Williams, J., Hirtle, M., Baume, C., Ikingura, J., Niaré, A., et al., 2010, 'Training needs assessment in research ethics evaluation among research ethics committee members in three African countries: Cameroon, Mali and Tanzania', Developing World Bioethics 10(2), 88-98.

Centers for Disease Control and Prevention, 2014, Tuskegee Study Timeline, viewed 9 December 2014, from http://www.cdc.gov/tuskegee/timeline.htm

Council on Health Research for Development, 2008, Cameroon: Alignment and Harmonization in Health Research, viewed 10 December 2014, from http://www. cohred.org/downloads/cohred_publications/Cameroon_web.pdf

Council for International Organizations of Medical Sciences, 2002, 'International ethical guidelines for biomedical research involving human subjects', Bulletin of Medical Ethics 182, 17-23.

Council on Health Research for Development, 2013, Health research and ethics workshop report, viewed 9 December 2014, from http://www.cohred.org/wpcontent/uploads/2011/05/SwazilandRECTrainingFinalReport.pdf

Data for African Development Working Group, 2014, Delivering on the data revolution in sub-Saharan Africa, viewed 30 March 2016, from http://www.cgdev.org/sites/ default/files/CGD14-01\%20complete $\% 20$ for $\% 20$ web $\% 200710$.pdf

Duncan, G.T., Elliot, M. \& Juan Jose Salazar, G., 2011, Statistical confidentiality: Principles and practice, Springer, New York.
Hugman, R., Pittaway, E. \& Bartolomei, L., 2011, "When "Do No Harm" is not enough: The ethics of research with eefugees and other vulnerable groups', British Journal of Social Work 41, 1271-1287.

Hyder, A.A., Wali, S.A., Khan, A.N., Teoh, N.B., Kass, N.E. \& Dawson, L., 2004, 'Ethical review of health research: A perspective from developing country researchers', Journal of Medical Ethics 30(1), 68-72.

Ijsselmuiden, C., Marais, D., Wassenaar, D. \& Mokgatla-Moipolai, B., 2012, 'Mapping African ethical review committee activity onto capacity needs; the March initiative And HRWEB's interactive database of RECs in Africa', Developing World Bioethics $12(2), 74-86$

Jeko, I., Mangwaya, E. \& Blignaut, S., 2012, 'Obtaining informed consent in nonwestern contexts: Reflections on fieldwork experiences in Zimbabwe', Acto Academica 44(4), 184-201.

Kass, N.E., Hyder, A.A., Ajuwon, A., Appiah-Poku, J., Barsdorf, N., Elsayed, D.E., et al , 2007, 'The structure and function of research ethics committees in Africa: A case 2007,' The structure and function of research ethics committees in Africa: A case
study', PLoS Medicine 4(1), E3, viewed 10 December 2014, from http://www.ncbi. $\mathrm{nlm}$.nih.gov/pmc/articles/PMC1779815

Kirigia, J.M., Wambebe, C. \& Baba, A., 2005, 'Status of National Research Bioethics Committees in the WHO African Region', BMC Medical Ethics 6, E10.

National Commission for the Protection of Human Subjects of Biomedical and Behavioral Research, 1979, The Belmont report: Ethical principles and guidelines for the protection of human subjects of research, viewed 10 December 2014, from http://www.fda.gov/ohrms/dockets/ac/05/briefing/2005-4178b_09_02_ Belmont $\% 20$ Report.pdf

Ndebele, P., Wassenaar, D., Benatar, S., Fleischer, T., Kruger, M., Adebamowo, C., et al., 2014, 'Research ethics capacity building in sub-Saharan Africa: A review of NIH Fogarty-funded programs 2000-2010', Journal of Empirical Research on Human Research Ethics 9, 24-40.

Office for Human Research Protections, U.S. Department of Health and Human Services, 2014, International compilation of human research standards, viewed 6 January 2015, from http://www.hhs.gov/ohrp/international/ intlcompilation/2014intlcomp.pdf

Presidential Commission for the Study of Bioethical Issues, 2011, Moral science: Protecting participants in human subjects research, Washington, D.C. viewed 10 December 2014, from http://bioethics.gov/node/558

Regional Reference Strategic Framework, 2006, 'Strategic framework for statistical capacity building in Africa', African Statistical Journal 2, 131-134, viewed 10 December 2014, from http://www.afdb.org/fileadmin/uploads/afdb/ Documents/Publications/African.Statistical.Journal_Vol2_2.Articles_6. ReferenceRegionalStrategicFramework.pdf

United Nations, 1948, Universal declaration of human rights, viewed 9 December 2014, from http://www.ohchr.org/en/udhr/documents/udhr_Translations/ eng. pdf

Weindling, P., 2001, 'The origins of informed consent: The international scientific commission on medical war crimes, and the Nuremberg code', Bulletin of the History of Medicine 75(1), 37-71.

World Medical Association, 2008, 'World Medical Association declaration of Helsinki: Ethical principles for medical research involving human subjects', Journal of the American Medical Association 310(20), 2191-1194.

Zuch, M., Mason-Jones, A.J., Mathews, C. \& Henley, L., 2012, 'Changes to the law on consent in South Africa: Implications for school-based adolescent sexual and reproductive health research', BMC International Health and Human Rights 12(3), viewed 10 December 2014, from http://www.biomedcentral.com/1472-698X/12/3 